\title{
Characterizations of MacNeille completions and topological functors
}

\section{Hans-E. Porst}

\begin{abstract}
In this note we give external characterizations of absolutely topological functors $U: A \rightarrow X$ (where $A$ may be a large category) as functors which are injective with respect to all functors based on the category $X$, or equivalently, which do not admit essential extensions over $X$. From this we deduce several characterizations and properties of the MacNeille completion of faithful functors. These results generalize the work of Horst Herrlich [Math. Z. 150 (1976), 101-110] in case of small categories, where the crucial point of this generalization lies in the fact that we cannot make use of the existence of MacNeilie completions and we therefore have to introduce a local-initialcompletion-construction.
\end{abstract}

\section{Introduction}

Considering a partly ordered set as a faithful (and amnestic) functor $U: A \rightarrow 1$, the MacNeille completion of this ordered set is a functor $E: A \rightarrow B$ over the category 1 which can be characterized categorically [3], [7]. In [7] Herrlich has generalized recently the notion of MacNeille completions to arbitrary faithful (and amnestic) functors $U: A \rightarrow X$, and shown that MacNeille completions may be characterized in this general situation just as in case $X=1$, if $A$ is a small category. This

Received 21 December 1977. Communicated by G.C.L. Brümmer. The author is indebted to Professor Brümer for encouraging him to publish this paper, and also acknowledges a helpful suggestion by Professor J.W. Gray. 
smallness condition is an important assumption, because the proofs make use of the existence of MacNeille completions, and MacNeille completions do not exist in general unless $A$ is small [7].

Since the MacNeille completion of a faithful functor $U: A+X$ is the smallest initial completion of $U$ (that is, extension of $U$ to a topological functor $V: B \rightarrow X$, see Section 1) which may be available, characterizations of MacNeille completions might be useful in searching for mild conditions for the existence of initial completions in case $A$ is a large category. For the importance of this problem we refer to [1], [2], $[7],[8]$.

By the same argument as above, Herirlich's proofs of the characterizations of absolutely topological functors [6] and initially and finally dense functors [7] are not transferable to functors $U: A \rightarrow X$ if $A$ is 1arge.

In this note we show that nevertheless all these characterizations hold with respect to arbitrary faithful functors, and show how these characterizations work practically. Moreover we distinguish absolutely topological functors $U: A \rightarrow X$ not only relative to faithful $X$-based functors (as in [7]) but also to arbitrary ones (as was suggested to the author by J.W. Gray). Further results are proved on the uniqueness, the existence, and some additional properties of MacNeille completions, which are not known as yet, or which could be proved only using special constructions of MacNeille completions.

Some historical remarks on the topic of this note seem to be necessary. External characterizations of topologicity related to those given here had first been given by Brümmer and Hoffmann in 1975 [5], using different methods in their proofs. Their results were unknown to the author when he presented the contents of this note at the Sussex Conference on Categories in 1976 [9]. Furthermore the author learned that his construction of a locally initial completion used in the proof of Theorem 1 had already been known to Brümmer and Hoffmann in 1975 - though not stated in [5].

\section{Preliminaries}

A pair $(A, U)$, where $U: A \rightarrow X$ is a functor, is called a category 
over $X .(A, U)$ is called concrete if $U$ is faithful; $(A, U)$ is called initially complete if $U$ is absolutely topological in the sense of [6]. A functor over $X, F:(A, U) \rightarrow(B, V)$ is any functor $F: A \rightarrow B$ with $V F \simeq U ; F$ is called an extension (of $(A, U)$ ) if $F$ is full and faithful. An extension $E:(A, U) \rightarrow(B, V)$ is called initial completion (of $(A, U))$ if $(B, V)$ is initially complete. Furthermore we need the following definitions.

DEFINITION 1. A category $(A, U)$ over $X$ is called injective if, for any extension $E:(B, V) \rightarrow(C, W)$ and any functor $E:(B, V) \rightarrow(A, U)$, there exists a functor $G:(C, W) \rightarrow(A, U)$ with $G E=F . \quad(A, U)$ is called c-injective if all involved categories are concrete. An injective extension is an extension $E:(\mathrm{A}, U) \rightarrow(\mathrm{B}, V)$ where $(B, V)$ is injective.

DEFINITION 2. Let $(B, V)$ be concrete. A functor $F:(A, U) \rightarrow(B, V)$ over $X$ is called essential, if any functor $G:(B, V) \rightarrow(C, W)$ such that $(C, W)$ is concrete and $G F$ is full, is a full functor itself.

DEFINITION 3. A functor $F:(A, U) \rightarrow(B, V)$ is called initialzy dense (dually, finally dense), if for any $B$-object $B$ there exists a $V$-initial source $(B,(B \stackrel{a}{\longrightarrow} F A))$ where $a$ ranges over some class of B-morphisms from $B$ to objects of the form $F A$.

The notion of initial denseness generalizes the notion of meetdenseness in the category of ordered sets [7]. It may be illustrated by the following examples.

1. The full embedding of the category Ind of indiscrete spaces into the category Top of topological spaces is neither initially dense nor finally dense.

2. The full embedding of the category Comp of compact Hausdorff spaces into the category $K$ of Kelley spaces is finally dense.

3. The full embedding of Comp into the category LocComp of locally compact Hausdorff spaces is initially and finally dense.

REMARK 1 . If $(B, V)$ is concrete, then $F:(A, U) \rightarrow(B, V)$ is initially dense, iff any source $(B,(B \stackrel{a}{\rightarrow} F A))$, where $a$ ranges over 
all B-morphisms from $B$ to all objects of the form $F A$, is V-initial.

REMARK 2. Any dense (codense) functor $F:(A, U) \rightarrow(B, V)$ (in the usual sense) is finally dense (initially dense) if $B$ is initially complete.

REMARK 3. $I_{A}:(A, U) \rightarrow(A, U)$ is initially and finally dense.

REMARK 4. If $(A, U)$ is $(c-)$ injective, then any extension of $(A, U)$ has a left inverse, and any essential extension of $(A, U)$ is an equivalence.

REMARK 5. Let $E:(A, U) \rightarrow(B, V)$ and $E^{\prime}:(B, V) \rightarrow(C, W)$ be extensions; if $E^{\prime} E$ is essential, then $E^{\prime}$ is essential.

\section{A characterization of initially complete categories}

Let us first point out the connection between the notions of essentiality and denseness, which will be useful in the following.

LEMMA 1. Let ( $B, V)$ be concrete; then any full and essential functor $E:(A, U) \rightarrow(B, V)$ is initially and finally dense.

Proof. Let us assume that there exists a source $\left\{B_{0},\left\{B_{0} \stackrel{a}{\longrightarrow} E A\right\} \mid A \in\right.$ ob $\left.A, a \in \mathrm{B}\left(B_{0}, E A\right)\right\}$ which fails to be $V$-initial. We construct a functor $G:(B, V) \rightarrow(C, W)$ such that $G E$ is full, but $G$ is not. For this let $C$ be the category with the same objects as $B$ and the following sets of morphisms (which are thought to be disjoint):

$$
C\left(B, B_{0}\right)=\left\{V B \stackrel{f}{\longrightarrow} V B_{0} \in \operatorname{mor} X / f+V a \text { for all } B_{0} \stackrel{a}{\longrightarrow} E A\right\} \text {, }
$$

where $f \downarrow V a$ iff there is a B-morphism $g: B \rightarrow E A$ such that the following diagram commutes:

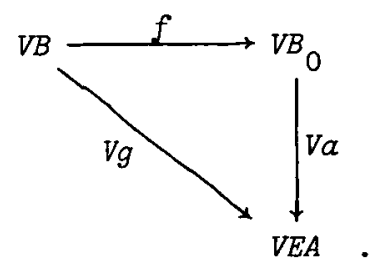

For $B^{\prime}=B_{0}$ we define 


$$
\mathrm{C}\left(B, B^{\prime}\right)=V\left(\mathrm{~B}\left(B, B^{\prime}\right)\right) \cup\left\{V b \cdot f \mid f \in \mathrm{C}\left(B, B_{0}\right), b \in \mathrm{B}\left(B_{0}, B^{\prime}\right)\right\} .
$$

Composition in $C$ is the composition in $X$. Then it is a (long but) straightforward calculation to see that $C$ is a category. With the following definitions we get a concrete category $(C, W)$ over $X$ and a functor $G:(B, V) \rightarrow(C, W)$ over $X:$

$$
W B:=V B, W f:=f \text {, and } G B:=B, \quad G_{f}:=V f \text {. }
$$

The definition of $\mathrm{C}$ implies the equation $\mathrm{C}\left(E A, E A^{\prime}\right)=V\left(\mathrm{~B}\left(E A, E A^{\prime}\right)\right)$. Hence $G E$ is full, because $E$ is full by assumption. Furthermore it is an immediate consequence of the construction that $G$ is not full. //

LEMMA 2. Let $(B, V)$ be conorete; then any initially and finally dense functor $E:(A, U) \rightarrow(B, V)$ is essential.

Proof. Let $G:(B, V) \rightarrow(C, W)$ be a functor over $X$, where $(C, W)$ is concrete and $G E$ is full, and let $f: G B \rightarrow G B^{\prime}$ be a C-morphism. Consider the family of morphisms $G E A \stackrel{G a}{\longrightarrow} G B \stackrel{f}{\longrightarrow} G B^{\prime} \stackrel{G a^{\prime}}{\longrightarrow} G E A^{\prime}$, where $A$ and $A^{\prime}$ range over ob $A$ independently and $a$ (respectively $a^{\prime}$ ) over all morphisms $E A \rightarrow B$ (respectively $B^{\prime} \rightarrow E A^{\prime}$ ). Apply $W$ to these morphisms and use the equivalence $W G \simeq V$ to deduce from the fullness of $G E$ and the denseness properties of $E$, that there is a B-morphism $f: B \rightarrow B^{\prime}$ with $G f=f \cdot \quad / /$

These lemmas yield the following proposition, which generalizes a similar result of [7] on small categories.

PROPOSITION 1. Let $E:(A, U) \rightarrow(B, V)$ be con extension, where $(B, V)$ is a concrete category over $X . E$ is essential iff $E$ is initially and finally dense.

The next proposition will yield a useful property of MacNeille completions; for a similar result see [8].

PROPOSITION 2. Full and essential functors preserve initial and final structures.

For the proof use the following lemma and its dual.

LEMMA 3. Let $E:(A, U) \rightarrow(B, V)$ be full and finally dense. Any B-source $\left(E A_{0},\left(E A_{0} \stackrel{E g_{i}}{\longrightarrow} E A_{i}\right)\right\}$ is V-initial, if the A-source 
$\left(A_{0},\left(A_{0} \stackrel{g_{i}}{\longrightarrow} A_{i}\right)\right\}$ is U-initial.

Consequentzy, if $\left(A_{0},\left(A_{0} \stackrel{g_{i}}{\longrightarrow} A_{i}\right)\right\}$ is a U-initial lifting of $\left\{x,\left(x \stackrel{f_{i}}{\longrightarrow} U A_{i}\right)\right\}$, then $\left(E A_{0},\left(E A_{0} \stackrel{E g_{i}}{\longrightarrow} E A_{i}\right)\right\}$ is a V-initial lifting of $\left\{x,\left(X \stackrel{f_{i}}{\longrightarrow} U A_{i} \simeq V E A_{i}\right)\right\}$.

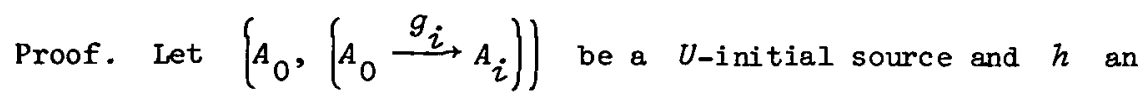
$X$-morphism such that

$$
V B \stackrel{h}{\longrightarrow} V E A_{0} \stackrel{V E g_{i}}{\longrightarrow} V E A_{i}=V\left(B \stackrel{h_{i}}{\longrightarrow} E A_{i}\right) \text {. }
$$

Then for each B-morphism $a: E A+B$ there is an A-morphism $h_{i}^{\prime}: A \rightarrow A_{i}$ such that $V E g_{i} \cdot h \cdot V a=V E h_{i}$. Hence the X-morphism $h_{a}: U A \rightarrow U A A_{0}$ corresponding to $h \cdot V a$ by the equivalence $U \simeq V E$ is of the form $h_{\alpha}=U h_{a}$, since $\left(A_{0},\left(A_{0} \stackrel{g_{i}}{\longrightarrow} A_{i}\right)\right\}$ is $U$-initial. Now the equation $V E h_{a}=h V a$ implies the existence of a B-morphism $\mathrm{h}: B+E A_{0}$ with $V h=h$, since $E$ is finally dense. $/ /$

The following theorem is essentially known for small categories [7].

THEOREM 1. Let $(A, U)$ be a category over $X$. The following assertions are equivalent:

(i) (A,U) is initially complete;

(ii) $(A, U)$ is concrete and injective;

(iii) $(A, U)$ is c-injective;

(iv) up to equivalence, $I_{A}$ is the only essential extension of $(A, U)$.

Proof. For the implication (i) $\Rightarrow$ (ii) use Herrlich's proof [7] with the obvious modification, and the fact that topological functors are faithful. 
$(i i) \Rightarrow(i i i) \Rightarrow(i v)$. Obvious by the remarks of Section 1 .

For the crucial implication $(i v) \Rightarrow$ (i), let us assume that there exists a source $\left\{X,\left(X \stackrel{f_{i}}{\longrightarrow} U A_{i}\right) \mid i \in I\right\}$ in $X$ which does not admit an $U$-initial lifting. We define a category $B$ as follows, where the morphism sets again are thought to be disjoint:

$$
\text { ob } B:=\text { ob } A \cup\{X\} \text {; }
$$

and for $B, C \in$ ob $\mathrm{A}$,

$\mathbf{B}(B, C):=U(\mathbf{A}(B, C))$,

$B(B, X):=\left\{f: U B \rightarrow X \mid f+f_{i}\right.$ for all $\left.i \in I\right\}$,

$B(X, C):=\{h: X \rightarrow U C \mid f \downarrow h$ for all $f \in B(B, X), B \in$ ob $A\}$,

$\mathrm{B}(X, X):=\{g: X \rightarrow X \mid f \downarrow g$ for all $f \in \mathrm{B}(B, X), B \in$ ob $\mathrm{A}$, and $g+f_{i}$ for all $\left.i \in I\right\}$.

Composition in $B$ is the composition in $X$. To show that $B$ is a category, one uses the following observations:

$$
\begin{aligned}
& \text { (i) } f_{i} \in \mathrm{B}\left(X, A_{i}\right) \text {; } \\
& \text { (ii) } \mathbf{B}(X, X)=\{g: X \rightarrow X \mid f+g \text { for all } f \in \mathbf{B}(B, X), B \in \text { ob } A\} \\
& =\left\{g: X \rightarrow X \mid g+f_{i} \text { for all } i \in I\right\} \text {. }
\end{aligned}
$$

We here use $g \downarrow f_{i}$ in the following obvious sense: there exists $g \in \mathrm{B}\left(X, A_{i}\right)$ such that $f_{i} g=\mathrm{g}$.

With the same definitions as in the proof of Lerma 1 we get a faithful functor $V: B \rightarrow X$ and a functor $E:(A, U) \rightarrow(B, V)$ which obviously is an extension. It is an immediate consequence of the definition of $B$ that $E$ is initially and finally dense. Furthermore, it is clear that $B$ admits a V-initial lifting of the source $\left(X,\left(X \stackrel{f_{i}}{\longrightarrow} U Y_{i}=V Y_{i}\right)\right)$; hence $E$ can not be an equivalence.

\section{Characterizations of MacNeille completions}

In this section all categories over $X$ are assumed to be concrete. A 
MacNeille completion is, by definition, an initial completion which, in addition, is initially and finally dense. As is shown by Herrlich [7], each initial completion $E:(A, U) \rightarrow(B, V)$ has a factorization $(A, U) \stackrel{E^{*}}{\longrightarrow}\left(B^{*}, V^{*}\right) \rightarrow(B, V)$, where $E^{*}$ is a MacNeille completion. Moreover we can show, axiomatically, that MacNeille completions are unique up to equivalence.

PROPOSITION 3. If $E:(\mathrm{A}, U) \rightarrow(\mathrm{B}, V)$ and $E^{\prime}:(\mathrm{A}, U) \rightarrow\left(\mathrm{B}^{\prime}, V^{\prime}\right)$ are MacNeille completions of $(A, U)$, then there is an equivalence $G:(B, V) \mp\left(B^{\prime}, V^{\prime}\right)$ such that $G E=E^{\prime}$.

Proof. The desired $G$ exists, since $\left(B^{\prime}, V^{\prime}\right)$ is injective. $G$ is an extension, because $E$ is essential, and $G$ is essential, since $E^{\prime}$ is essential. Now use Remark 4. //

As in the case of small categories we now get the following characterization of MacNeille completions.

THEOREM 2. If $E:(A, U) \rightarrow(B, V)$ is an extension, then the following assertions are equivalent:

(i) $E$ is a MacNeille completion of $(A, U)$;

(ii) $E$ is essential and injective;

(iii) $E$ is the smallest injective extension of $(A, U)$ (that is, if $E^{\prime}:(A, U) \rightarrow(C, W)$ is an injective extension, then there is an extension $G$ of $(B, V)$ such that $\left.G E=E^{\prime}\right)$;

(iv) $E$ is a minimal injective extension of (A, U) (that is, if $E$ has a factorization $E=G E^{\prime}$ over an injective extension $E^{\prime}$ such that $G$ is an extension, then $G$ is an equivalence);

(v) $E$ is a maximal essential extension.

Moreover any of these conditions imply

(vi) $E$ is the largest essential extension.

Proof. By the preceding remarks and results most implications are obvious. For the implications $(i i i) \Rightarrow(i i),(i v) \Rightarrow$ (ii), observe that in both cases $E$ is equivalent to the MacNeille completion contained in it. 
Note that the extensions are not really "ordered".

REMARK. The implication $(v i) \Rightarrow(i i)$ is valid, for example, if MacNeille completions exist (see [3]).

COROLLARY 1. If $E:(A, U) \rightarrow(B, V)$ is a MacNeille completion, then $E$ preserves initial and final structures.

COROLLARY 2. A MacNeille completion of a concrete category $(A, U)$ is an equivalence if and only if $(A, U)$ is initially complete.

COROLLARY 3. If $(A, U) \rightarrow(B, V)$ is an extension and if the MacNeille completion of $(B, V)$ exists, then the MacNeille completion of $(A, U)$ exists and admits an extension to the MacNeille completion of $(B, V)$.

COROLLARY 4. Let $E:(A, U) \rightarrow(B, V)$ be an essential extension. If one of these categories has a MacNeille completion, then so has the other, and both MacNeille completions are equivalent.

\section{Applications and remarks}

The characterization of absolutely topological functors $U: A \rightarrow X$ as concrete and injective pairs $(A, U)$ can be used to get very easily some of the standard results. For example the well known duality property, or the fact that full topological functors are equivalences [6], are immediate consequences. It also works practically as a checking criterion for initial completeness: the category Comp fails to be initially complete, because Comp $\hookrightarrow$ LocComp is a proper essential extension; moreover both categories have (topological) MacNeille completions in Top and these are equivalent

It should be mentioned that all assertions of this note remain valid with respect to the "strong" notion of initial completeness [7] (that is, the $U$-initial liftings are to be exact), provided we replace "faithful" by "faithful and amnestic", "extension" by "full embedding", and "equivalence" by "isomorphism". Similarly one can consider fibre small categories over $X$ only, and so one gets statements as, for example, $"(A, U)$ is a Top-category over $X$ iff $(A, U)$ is concrete, fibre small, and infective relative to E.ll (fibre small) categories over $X$ ".

Finally we remark that the notion of essentiality seems to be fruitful 
with respect to questions on the existence of initial completions, although the notion of injectivity looks more elegant.

\section{References}

[1] Philippe Antoine, "Etude élémentaire des catégories d'ensembles structurés" (Première partie), Buzz. Soc. Math. Bezg. 18 (1966), $142-164$.

[2] Philippe Antoine, "Êtude élémentaire des catégories d'ensembles structurés" (Deuxième partie), Bulz. Soc. Math. Belg. 18 (1966), $387-414$.

[3] B. Banaschewski and G. Bruns, "Categorical characterization of the MacNeille completion", Arch. Math. (Basel) 18 (1967), 369-377.

[4] G.C.L. Brümmer, "Topological functors and structure functors", Categorical topology, 109-135 (Proceedings of the Conference held at Mannheim, 1975. Lecture Notes in Mathematics, 540 . SpringerVerlag, Berlin, Heidelberg, New York, 1976).

[5] G.C.L. Brümmer and R.-E. Hoffmann, "An external characterization of topological functors", Categorical topology, 136-151 (Proceedings of the Conference held at Mannheim, 1975. Lecture Notes in Mathematics, 540. Springer-Verlag, Berlin, Heidelberg, New York, 1976).

[6] Horst Herrlich, "Topological functors", General Topology and Appl. 4 $(1974), 125-142$.

[7] Horst Herrlich, "Initial completions", Math. 2. 150 (1976), 101-110.

[8] H. Herrlich and L.D. Nel, "Cartesian closed topological hulls", Proc. Amer. Math. Soc. 62 (1977), 215-222.

[9] Hans-E. Porst, "On the MacNeille completion of faithful functors", Category theory meeting, Isle of Thorns, Sussex, England (Mimeographed Abstracts, 1976).

Fachsektion Mathematik, Universität Bremen,

Bremen, Germany. 